\title{
Towards open science: what we know and what we need to know
}

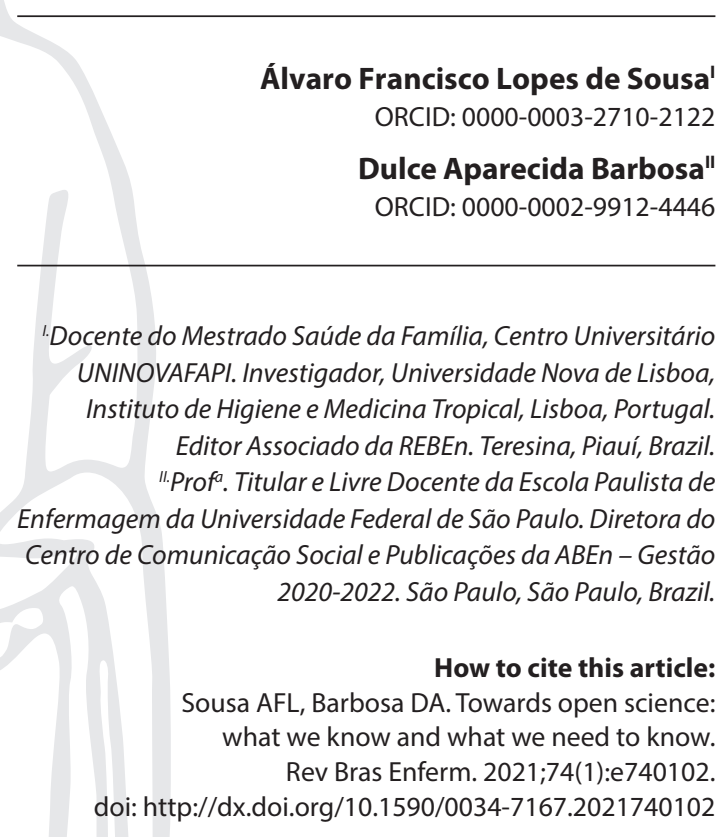
doi: http://dx.doi.org/10.1590/0034-7167.2021740102
Open science presents itself as a set of policies and actions to disseminate research results in an accessible, free and reusable and reproducible way through public digital repositories. As a movement, it uses three basic elements: open access to publications; data opening (whether raw, models, specifications, or documentation); computational process opening (software and algorithms) ${ }^{(1)}$.

Although it is not a new phenomenon, the term can still cause strangeness even to experienced researchers. Open access to articles, as the first element, encountered (and still finds) great resistance to becoming unanimous, although pressure from the scientific society and funding agencies has accelerated the progress of this stage. On the other hand, data opening seems to have been better received, at least in its interface related to the deposit of scientific manuscripts in the preprint format, however this is only the beginning.

Concerning the Brazilian experience, SciELO and the Brazilian Institute of Information in Science and Technology (IBICT - Instituto Brasileiro de Informação em Ciência e Tecnologia) have been leading the opening process and for some time have designed guidelines and strategies to guide their journals towards open science: TOP (Transparency and Openness Promotion) (2). This system interestingly presents levels of openness experimentation that range from pointing out what is a certain item to making it conditional on it being expressly fulfilled for the manuscript to be published.

Although it has existed since 2017, it was only in 2020 that the alignment of Brazilian journals to TOP was indeed accelerated, and significant changes will be adopted in the journals in the coming months and years to adapt to such principles.

Having this information and basing ourselves on the fact that historically changes have been the target of resistance, especially when they happen in an ancient system, like the scientific publication system, we use our privilege to take on multiple roles (author, reviewer, and editor) among the scientific publication process in Brazilian journals to reflect and point out in this editorial four central issues related to editorial management that should be recurrent among the actors involved in the publication process in the coming years months:

\section{What changes can the opening of the peer review process impose?}

The Open Peer Review (OPR) is undoubtedly among the closest changes, the one that leads to numerous questions, causing further developments even if the initial recommendation is that the magazines experience progressive levels of openness.

The international experience of groups such as BioMed Central (BMC) and the Multidisciplinary Digital Publishing Institute (MDPI) reveals that vertically enforcing fully open peer review can be harmful to the process at some level. In these publishers, the OPR model led to immediate repercussions in the review process, with more researchers refusing to perform this task. This consequence meant that, after 20 years of fully open peer review, the BMC group started to adopt transparent peer review, in which it offers reviewers the possibility to identify themselves. 
2. If the fully open peer review is standard, do reviewers become co-responsible for the published material?

Article blind validation has always limited the consequences of mistaken acceptance of manuscripts that are not as rigorous as necessary. However, this seems to be with the days numbered. Open science allows access to research notebooks, databases and even previous records of research protocols; therefore, reviewers' responsibility for the published material will be inevitable. In this sense, "post-publication" assessment, whether in the form of letters to the editor, comments on preprint servers, or others, should become more frequent since readers can compare the published version with authors' initial claims.

On the other hand, the implementation of open science should impose a reduction in the practice of slicing studies (Salami Science) due to the requirement to deposit previous records of studies, allowing identifying multiple studies derived from a single database.

\section{What are the implications of open science for author- ship criteria?}

Another aspect that we must consider is authorship criteria, since, to date, there is no (and should not be) orientation of the repositories of what factors should be considered when listing the authors. Another detail refers to the restriction in the number of authors that some journals use (usually from six to ten authors) and which is not a concern of preprints.
In the absence of a clear directive, we suggest that authors follow the classic guidelines of authorship criteria used in the health field already in preprint submission ${ }^{(3)}$, considering a journal's permissions and norms about it. We suggest that the list of preprint authors should be the same as that of the article and in the same sequence, since adding or subtracting authors in the preprint version may be considered misconduct.

\section{When and how to use texts deposited on preprint servers?}

The number of journals that allow assessment of texts previously deposited in preprint servers is increasingly frequent as well as allowing authors to cite such texts in their references. However, this should be done with caution, assessing the quality of the server where the text was published (it is suggested to use non-commercial servers that use DOI (Digital Object Identifier)). Authors should pay attention and ensure that their text is deposited under a valid license ${ }^{(4)}$ and that allows the use and reuse of preprints, an error that has been common in the BiorXiv server.

It is also extremely important that authors pay attention to the different versions of the same text deposited in preprint format and that they quote the version to which they refer, since the same text can have several versions with severe changes within the preprint platforms.

The central aspects raised in this editorial can lead researchers to debate about this new way of doing science, considering that it is the path set for the academic community.

\section{REFERENCES}

1. Anglada L, Abadal E. "¿Qué es la ciencia abierta?". An ThinkEPI. 2018;12:292-8. doi: 10.3145/thinkepi.2018.43

2. Aalbersberg IJ, Appleyard T, Brookhart S, Carpenter T, Clarke M, Curry S, et al. Making science transparent by default: introducing the TOP statement. OSF Preprints. 2018. doi: 10.31219/osf.io/sm78t

3. International Committee of Medical Journal Editors (ICMJE). Recommendations [Internet]. 2021[cited 2021 Jan 20]. Available from: http:// www.icmje.org/recommendations/browse/

4. McKenzie L. Biologists debate how to license preprints. Nature. 2017. doi: 10.1038/nature.2017.22161 